change between high- and low-predation populations, and whether selection against brightly coloured male offspring is so great in high-predation populations that the linkage disequilibrium brought about by assortative mating by preferential females can never get established. We acknowledge limitations to our study, but reiterate that it provides strong evidence that selection against the attractive male character has led to correlated changes in female preference, as predicted by Fisher's runaway process of sexual selection.

Felix Breden Gregory StONER Division of Biological Sciences, University of Missouri, Missouri 65211, USA

1. Houde, A.E. Nature 333, 711 (1988).

2. Breden, F. \& Stoner, G. Nature 329, 831-833 (1987)

3. Endler, J.A. Nature 332, 593-594 (1988)

4. Breden, F. Nature 332, 594 (1988).

5. Stoner, G. \& Breden, F. Behav. Ecol. Sociobiol. 22, 285291 (1988).

\section{Mycorrhizal infection and plant species diversity}

SIR-Grime et al. ${ }^{1}$ argue that the presence of mycorrhizas leads to an increase in the plant species diversity of laboratory microcosms. They grew species-rich mixtures of plants with and without infection by mycorrhizal fungi, and found a small but significant increase in plant species diversity as a result of mycorrhizal infection. The mechanism they invoke involves "the improved yields of the subordinate species [which] were not simply due to the reduced vigour or increased root/shoot biomass of the infected canopy dominant". The small, suppressed plants are assumed to obtain carbohydrate from the larger, dominant plants via shared mycorrhizal hyphae. We take issue with this and propose a simpler explanation.

One criticism is that the radiolabelled $\mathrm{CO}_{2}$ studies $^{2.3}$ cited by Grime et al. as showing net carbohydrate flow into suppressed plants, in fact show carbohydrate movement only between plants. We agree there is clear evidence of carbon transfer through the mycorrhizal network, but not that there is a net flow of carbon to suppressed 'sink' plants. The obvious experiment of labelling $\mathrm{CO}_{2}$ in the subordinate plants has never been done.

Grime et al. suggest that the "export of assimilate from 'source' (canopy dominant) to 'sink' (understorey components) through a common mycorrhizal network may be an important element of the mechanism maintaining species-rich communities on infertile soils." We propose a simpler interpretation. Although 'diversity' increased in the mycorrhizal plots, species richness was identical under the two treatments; the increase in diversity results from a reduction in dominance following mycorrhizal infection. The dominant plant in both treatments (Festuca ovina) declined in shoot weight by more than $30 \%$ following infection, and clearly loses more than it gains from mycorrhizal association. A substantial part of the response in the diversity index can be attributed to this alone (of the total increase in diversity of $47 \%$, over half is accounted for by reduced dominance alone).

We suggest that the improved growth of previously suppressed species can be attributed to competitor release following mycorrhizal infection, coupled with the undisputed potential of mycorrhizae to enhance the uptake of phosphorus and nitrogen in nutrient-limited conditions. We see no need to invoke net carbohydrate flow into suppressed plants and would prefer to wait for direct evidence before accepting this more complicated explanation.

Furthermore, it is most unlikely that the result reported by Grime et al. is general; in this case mycorrhizal infection was detrimental to the growth of the dominant plant, but there will be others in which the dominant plant benefits from mycorrhizal association, and in becoming more dominant, brings about a reduction in plant species diversity.

J.M. BERGELSON Department of Zoology NJ-15,

University of Washington,

Seattle, Washington 98195, USA

M.J. Crawley Department of Pure and Applied Biology, Imperial College, Silwood Park, Ascot, Berks SL5 7PY, UK

Grime ET AL. RePLy-In grasslands on shallow calcareous soils, grazing has long been implicated as a control on plant species diversity, but the widespread occurrence of mycorrhizal infection in plants growing in such situations has been largely ignored. The objective of our experiment was to determine the impact of these two factors on the outcome of competitive interactions between species in microcosms. To examine effects arising from infection by mycorrhizal fungi, plant species known to differ in their susceptibility to infection were included, ranging from Rumex acetosa (not mycorrhizal) to Centaurium erythraea (highly infected). The year-long experiment indicated that infection promoted diversity and, in particular, increased the biomass of some of the subordinate species.

Bergelson and Crawley suggest that this treatment, like grazing, operated through selective debilitation of the dominant species, Festuca ovina. We agree that grazing and mycorrhizal infection are likely to have brought about competitive release, but Bergelson and Crawley seem to have overlooked features of the data which indicate effects peculiar to mycorrhizal infection, and not attributable to the reduced vigour of the dominant $F$. ovina.
In particular, the heavily mycorrhizal species $C$. erythraea showed a spectacular improvement in survivorship and yield when infected, but showed no evidence of competitive release when grazing reduced the yield of the dominant plant to levels considerably below those achieved in the mycorrhizal treatment. More telling still, inoculation with mycorrhizal fungi produced no effect on the yield of the nonmycorrhizal species $R$. acetosa.

We investigated possible contributory factors to the growth responses by feeding ${ }^{14} \mathrm{CO}_{2}$ to donor plants of $F$. ovina. Radioactivity levels were much higher in infected plants, providing, as we stated, support for earlier suggestions that export of assimilate through a common mycorrhizal network may be an important element of the mechanism maintaining diversity. We did not put the case more strongly because the experiment was not designed to determine net carbon movement. Earlier studies however, have shown that the magnitude of carbon transfer from potential donors to receivers can be influenced by shading of the receivers. This suggests that the polarity of movement through mycorrhizas is determined by source-sink relations and that the shaded subordinate plants in our experiment would receive more carbon than the well-illuminated dominants. The uninfected species $R$. acetosa supported this contention as it was the only one that failed to accumulate labelled carbon.

Furthermore, mycorrhizal infection is known to be a drain upon the carbon reserve of the host plant as carbon allocation to roots can increase by $10 \%$ on infection $^{4.5}$. Although such a drain is apparently sustained without adverse effect by dominant plants, it could be a problem for suppressed individuals, and it seems reasonable to consider that in such cases they are supplied with carbon by transfer from other individuals in the interconnected system.

We agree with Bergelson and Crawley that experiments to determine net flow of carbon to suppressed plants by labelling subordinate individuals are desirable. We have some simpler microcosms with single and two-species combinations which will enable such investigations to be made.

J. P. GRIME

J. M. MACKEY

S. H. HILLIER

D. J. READ

Unit of Comparative Plant Ecology

$(N E R C)$ and Department of Plant Sciences,

The University, Sheffield S102TN, UK

1. Grime, J.P., Mackey, J.M.L.. Hillier, S.H. \& Read, D.J. Nature 328, 420-422 (1987)

Francis, R. \& Read, D.J. N

Nature 307, 53-56(1984)

Read, D.J., Francis, R. \& Finlay, R.D. Ecological Inter actions in the Soil (Blackwell Scientific. Oxford, 1985).

4. Pang. P.C. \& Paul, E. A. Can. J. Soil Sci. 60. 241 (1980).

Snellgrove, R.C., Splitsoesser. W.E., Stribley. D.P. \& Tinker. P. B. New Phytol. 92, 75-81 (1982). 